\title{
Lizard Scale Patterns Described with Antiferromagnetic Model
}

\author{
The pattern of green and black scales on an ocellated lizard can be
} described with the two-parameter Ising model for antiferromagnetic systems.

By Erika K. Carlson

E ach scale of an ocellated lizard, a reptile found in southwestern Europe, can be either green or black. As a lizard matures, individual scales change from one color to the other, eventually forming a snaking, labyrinthine mosaic at adulthood. Michel Milinkovitch of the University of Geneva and colleagues have now found that the Ising model of antiferromagnetism can describe the process by which these scales settle into their final pattern [1].

Previously, the team modeled the development and distribution of the lizard's scales as a cellular automaton-a computational model in which cells on a grid evolve according to well-defined rules. But this model had 14 parameters.

Seeking a simpler description, the researchers considered the antiferromagnetic Ising model, in which atoms or molecules in a material flip between spin states so that neighboring particles tend to have opposite spins. This model has just two parameters: the interactions between neighboring particles

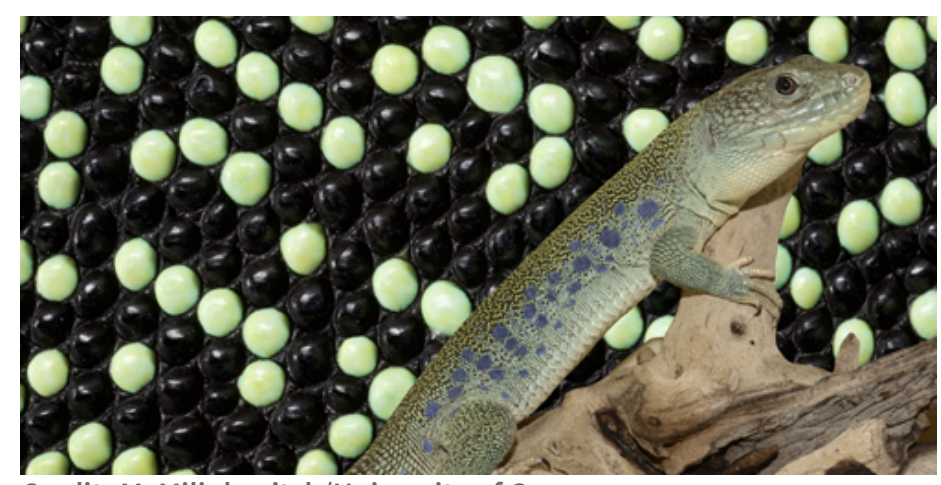

Credit: M. Milinkovitch/University of Geneva

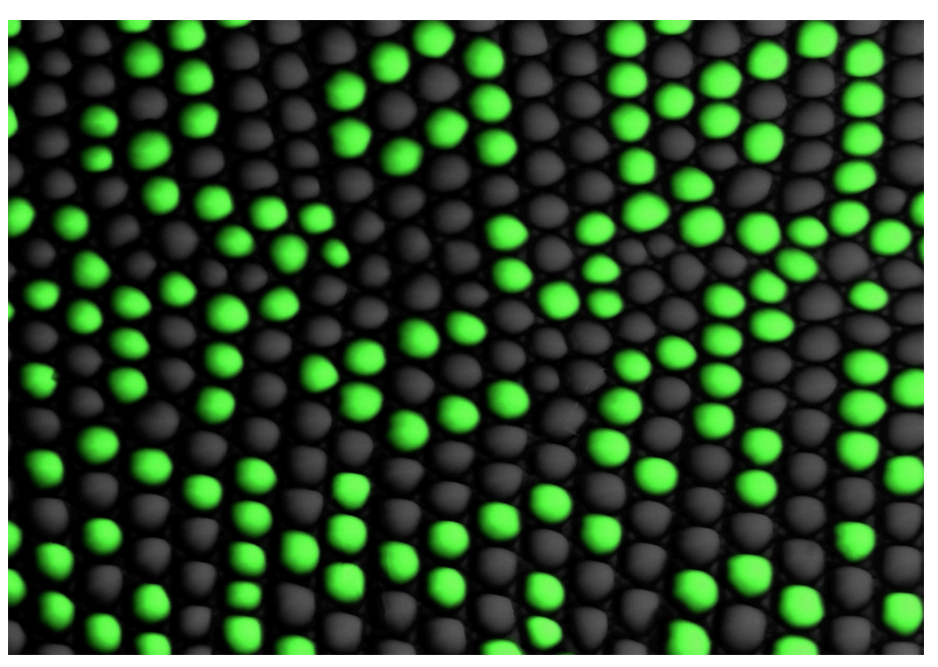

Video 1: The Ising model of antiferromagnetism accurately recreates the way an ocellated lizard's scales change color over time.

Credit: S. Zakany/University of Geneva

and the strength of an external magnetic field.

Milinkovitch and colleagues found that the antiferromagnetic Ising model accurately recreated the time evolution of these lizards' scale colors, the labyrinthine nature of their final patterns, and the predominant balance of green and black scales. In their model, the scales' tendency to avoid being the same color as too many of their neighbors was analogous to the interaction between spins in an antiferromagnet, while an external forcing analogous to a magnetic field generated a slight preference for black over green scales. The researchers 
wonder if natural selection led this species to favor its particular York City. pattern and balance of colors; to probe this question further, they plan to investigate other species with color-changing scales that form patterns over time.

Erika K. Carlson is a Corresponding Editor for Physics based in New

\section{REFERENCES}

1. S. Zakany et al., "Lizard skin patterns and the Ising model," Phys. Rev. Lett. 128, 048102 (2022). 